\title{
Correction to: Heavy-Tailed Kernels Reveal \\ a Finer Cluster Structure in t-SNE Visualisations
}

Dmitry Kobak, George Linderman, Stefan Steinerberger,

Yuval Kluger, and Philipp Berens

\section{Correction to: \\ Chapter "Heavy-Tailed Kernels Reveal a Finer Cluster Structure in t-SNE Visualisations" in: U. Brefeld et al. (Eds.): Machine Learning and Knowledge Discovery in Databases, LNAI 11906, https://doi.org/10.1007/978-3-030-46150-8_8}

The chapter was inadvertently published without the supplementary file. The supplementary file and its ESM Hint have been added to the chapter. 